\title{
Describing the indirect impact of COVID-19 on healthcare utilisation using syndromic surveillance systems
}

Claire F. Ferraro ${ }^{1 *}$, Lucy Findlater ${ }^{2}$, Roger Morbey ${ }^{3}$, Helen E. Hughes ${ }^{3}$, Sally Harcourt ${ }^{3}$, Thomas C. Hughes ${ }^{4}$, Alex J. Elliot ${ }^{3}$, Isabel Oliver ${ }^{1}$ and Gillian E. Smith ${ }^{3}$

\begin{abstract}
Background: Since the end of January 2020, the coronavirus (COVID-19) pandemic has been responsible for a global health crisis. In England a number of non-pharmaceutical interventions have been introduced throughout the pandemic, including guidelines on healthcare attendance (for example, promoting remote consultations), increased handwashing and social distancing. These interventions are likely to have impacted the incidence of non-COVID-19 conditions as well as healthcare seeking behaviour. Syndromic Surveillance Systems offer the ability to monitor trends in healthcare usage over time.
\end{abstract}

Methods: This study describes the indirect impact of COVID-19 on healthcare utilisation using a range of syndromic indicators including eye conditions, mumps, fractures, herpes zoster and cardiac conditions. Data from the syndromic surveillance systems monitored by Public Health England were used to describe the number of contacts with NHS 111, general practitioner (GP) In Hours (GPIH) and Out-of-Hours (GPOOH), Ambulance and Emergency Department (ED) services over comparable periods before and during the pandemic.

Results: The peak pandemic period in 2020 (weeks 13-20), compared to the same period in 2019, displayed on average a 12\% increase in NHS 111 calls, an 11\% decrease in GPOOH consultations, and a 49\% decrease in ED attendances. In the GP In Hours system, conjunctivitis consultations decreased by $64 \%$ and mumps consultations by $31 \%$. There was a $49 \%$ reduction in attendance at EDs for fractures, and there was no longer any weekend increase in ED fracture attendances, with similar attendance patterns observed across each day of the week. There was a decrease in the number of ED attendances with diagnoses of myocardial ischaemia.

Conclusion: The COVID-19 pandemic drastically impacted healthcare utilisation for non-COVID-19 conditions, due to a combination of a probable decrease in incidence of certain conditions and changes in healthcare seeking behaviour. Syndromic surveillance has a valuable role in describing and understanding these trends.

Keywords: Syndromic surveillance, Healthcare utilisation, Viral, Epidemiology, Coronavirus, Pandemic

\section{Background}

The coronavirus pandemic (COVID-19) is currently causing an unprecedented global health crisis with significant impact on health and social care services and

\footnotetext{
* Correspondence: claire.ferraro@nhs.net

${ }^{1}$ Field Service, UK Health Security Agency, Bristol BS1 6EH, UK

Full list of author information is available at the end of the article
}

society worldwide. As of 21st June 2021, there have been over 178 million cases and 3.8 million deaths from SARS-CoV-2 globally [1]. The first cases in England were identified at the end of January 2020 [2, 3]. Widespread transmission in the UK led to a rapid increase in incidence during March reaching an initial peak of new cases in England in April [4]. During 2020, the daily

C C The Author(s). 2021 Open Access This article is licensed under a Creative Commons Attribution 4.0 International License, which permits use, sharing, adaptation, distribution and reproduction in any medium or format, as long as you give appropriate credit to the original author(s) and the source, provide a link to the Creative Commons licence, and indicate if changes were made. The images or other third party material in this article are included in the article's Creative Commons licence, unless indicated otherwise in a credit line to the material. If material is not included in the article's Creative Commons licence and your intended use is not permitted by statutory regulation or exceeds the permitted use, you will need to obtain permission directly from the copyright holder. To view a copy of this licence, visit http://creativecommons.org/licenses/by/4.0/ The Creative Commons Public Domain Dedication waiver (http://creativecommons.org/publicdomain/zero/1.0/) applies to the data made available in this article, unless otherwise stated in a credit line to the data. 
number of deaths in England was at its highest at just over 1000 deaths in week 15 (6-12th April 2020).

The UK government's COVID-19 public health information campaign was launched in early February encouraging individuals to increase handwashing and improve respiratory hygiene to 'catch' coughs and sneezes [5]. On the 12th March, the government announced a move from 'contain' to the 'delay' phase [6]. The announcement advised the public to use a new online version of the National Health Service (NHS) remote telephone health advice line; NHS 111 [7]. Primary care (general practitioner; GP) services in England started to conduct remote consultations in the first instance [8]. Stepwise restrictions on social mixing and daily life were introduced over the subsequent two weeks until the 23rd March when the first national 'lockdown' tightened restrictions further (Fig. 1). Ambulance services and emergency departments (EDs) continued to operate as usual throughout; however, significant changes in healthcare seeking behaviour were observed, potentially due to changes in need for urgent healthcare, possible fears of contracting the infection in healthcare settings, and a desire to 'protect the NHS' [10].
Public Health England (PHE; please note - on 1 October 2021 PHE was replaced by UK Health Security Agency and the Office of Health Improvement and Disparities) publishes weekly, publicly available, surveillance reports for the monitoring of the COVID-19 pandemic in England [11]. The COVID-19 surveillance activities reported are carried out across multiple data sources including laboratory confirmed cases; community outbreaks; internet surveys and search activity; hospitalisations; and mortality. These surveillance activities are further supplemented by syndromic surveillance of healthcare utilisation across five different areas of the NHS: NHS 111 (calls and online assessments); GP consultations, both In Hours (scheduled) and Out-of-Hours (unscheduled); ambulance calls; and ED attendances.

'Syndromic' surveillance is "the process of collecting, analysing and interpreting health-related data to provide an early warning of public health threats which require public health action" [12]. It is powerful at detecting changes in relatively non-specific symptoms or preliminary diagnosis information collected during routine healthcare provision. This can offer the near real-time ability to provide early warning, improve situational

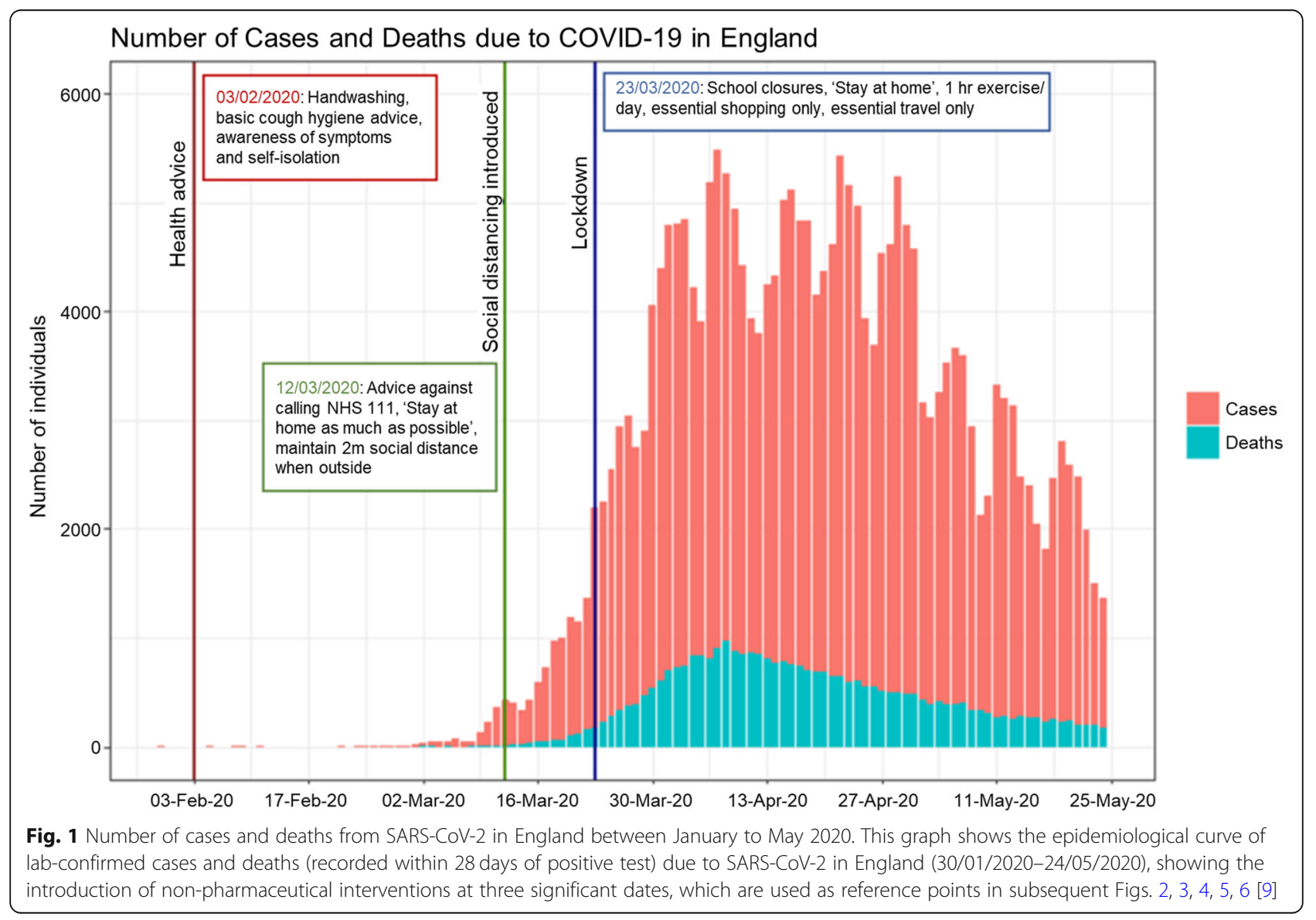


awareness, and monitor the emergence and spread of common infectious diseases and the public health impact of non-infectious diseases through the population. The important role of syndromic surveillance has been demonstrated during both major emergencies and mass gathering events, e.g. the 2009 global influenza pandemic $[13,14]$, during periods of extreme hot $[15,16]$ and cold [17] weather, and events such as the London 2012 Olympic and Paralympic Games [18]. COVID-19 has generated several new challenges for the syndromic surveillance systems, including: the impact of media reporting and social distancing measures on healthcareseeking behaviour, changes in healthcare system delivery, and an increased demand for rapid analysis and dissemination of findings [19].

Understanding changes in healthcare demand is essential to interpret surveillance information. The PHE syndromic surveillance systems monitor a wide range of indicators of illness and healthcare utilisation. This paper aims to assess the indirect impact of COVID-19 and the introduction of non-pharmaceutical interventions (NPIs) on healthcare utilisation across different aspects of the NHS using syndromic surveillance. The syndromic indicators, chosen a priori, aim to explore the impact of mitigation measures such as increased handwashing, social distancing, and lockdown on healthcare utilisation across the different systems. In addition, herpes zoster and cardiac conditions were chosen as indicators which may indicate changes in decision making thresholds for individuals' seeking healthcare during the pandemic.

\section{Methods}

\section{Data sources}

Data from the five PHE national syndromic surveillance systems were included in this study: remote health advice (NHS 111) calls and online assessments; GP In Hours consultations (GPIH); GP Out-of-Hours consultations (GPOOH); emergency department attendances (Emergency Department Syndromic Surveillance System (ED)); and ambulance calls [17]. The systems differ in their coding systems, range of indicators, and coverage across England (Table 1) [13, 21, 22]. The ED analysis was restricted to only those EDs which had reported throughout the study time period.

\section{Indicators}

This study explored the trends in several conditions during the COVID-19 pandemic, considered likely to be affected by different components of the NPIs implemented in England (Table 1). These conditions were selected a priori, having been previously defined as 'syndromic indicators', specific to each surveillance system:
1. Eye condition indicators, monitored through GPOOH, GPIH, and NHS 111, were chosen because the incidence of infective conjunctivitis has been known to reduce due to handwashing campaigns during previous pandemics [23].

2. The introduction of social distancing measures would likely have an impact on other highly transmissible infectious diseases and therefore, knowing there had been a recent national outbreak of mumps across England, this was chosen as an indicator, monitored through GPIH [24].

3. Attendances at EDs for fractures, which are severe enough to warrant attendance and therefore may be representative of true incidence, were chosen as a further indicator, as national lockdown restrictions are likely to have changed daily activities (for example, there might be fewer fractures associated with injuries occurring while commuting to work or playing team sports). Lockdown measures resulting in less travel, working from home, children not attending school and closure of the night-time economy, may have resulted in a change to the distribution in time, gender, and age-groups of those attending ED for fractures. Therefore, ED attendances for fractures during the pandemic, broken down by gender, were analysed by hour of the day and day of the week.

4. To investigate changes in healthcare utilisation, the indicator for herpes zoster was chosen, monitored through GPIH, as incidence of a re-emergent viral infection was considered unlikely to be significantly affected by the COVID-19 pandemic NPIs, but rather indicate changes in individuals' decision making threshold for seeking healthcare.

5. Cardiac indicators (chest pain/myocardial infarction indicator $(\mathrm{GPOOH})$, myocardial ischaemia (ED), and chest pain (ambulance calls)) were chosen as indicators of severe life-threatening conditions which would usually require healthcare, therefore demonstrating changes in healthcare seeking behaviour during the pandemic.

\section{Time period}

Trends of the selected indicators between Wednesday 1 st January 2020 to Sunday 24th May 2020 inclusive were compared visually with Tuesday 1st January 2019 to Friday 24th May 2019. Key dates when public health messages or other interventions were introduced were considered (Fig. 1). The nine-week (63-day) period of the first national lockdown (week 13 - week 22: Monday 23rd March 2020 to Sunday 24th May 2020) was chosen to compare epidemiological trends at the peak of the COVID-19 pandemic with the equivalent period, matched by day of the week, in 2019 (week 13 - week 22: Monday 25th March 2019 to Sunday 26th May 2019). 


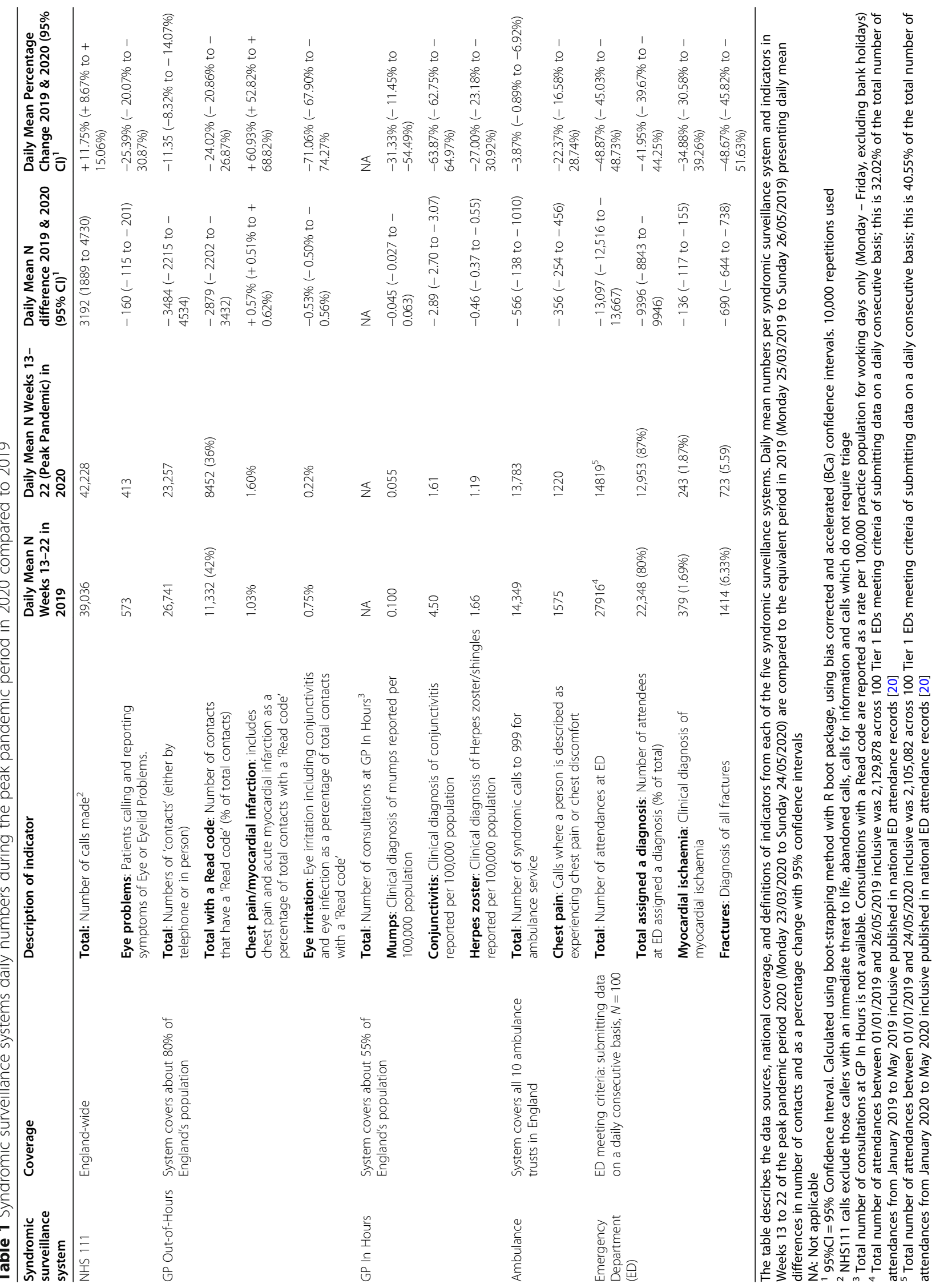




\section{Analysis}

Data on the number of total attendances and indicatorspecific attendances are presented differently for each syndromic surveillance system: as a rate per 100,000 registered population for GPIH; as the percentage of total calls for NHS 111; as counts for ambulance calls (for syndromic type calls only, not all ambulance calls) and ED; and as a percentage of total contacts with a 'Read code' for GPOOH. Read codes are a coded thesaurus of clinical terms which record findings and procedures in NHS settings [25]. Seven day moving averages (adjusted for bank holidays) were calculated for all systems. The GPIH seven day moving average additionally adjusts for the number of working days in each week [26].

To investigate the overall number of attendances, consultations, or contacts for each surveillance system over the period of interest, trends were visually inspected to describe the total numbers and seven day moving averages over time in 2020 compared to 2019, for NHS 111, GPOOH, ambulance calls, and ED (these data were not available for GPIH). Daily mean number of attendances, consultations, or contacts during the nine-week peak period in 2020 and 2019 (weeks 13 to 22) and the daily mean differences were calculated and confidence intervals estimated by bootstrap sampling $(10,000$ repetitions used) using the bias corrected and accelerated (BCa) methodology to minimise bias. Boot-strapping enabled estimation of confidence intervals for differences, including rates and counts, without requiring modelling assumptions about underlying probability distributions.

Plots were produced describing the total daily number and seven day moving average of reported calls or consultations for indicator conditions monitored by each system, for 2020 compared to 2019, to explore how their epidemiology changed throughout the COVID-19 pandemic.

All analyses were conducted in $\mathrm{R}$ (version 3.5.2) using tidyverse, boot, and ggplot2 packages [27-30].

\section{Results}

This study used PHE syndromic surveillance data from Wednesday 1st January 2020 to Sunday 24th May 2020, and Tuesday 1st January 2019 to Friday 24th May 2019 (one day fewer due to 2020 being a leap year). These figures represent the data routinely available through the syndromic surveillance systems, but for some systems this is not necessarily representative of the whole of England (Table 1). The total number of NHS 111 calls was $5,630,117$ for this time period in 2019 , and 6,175,249 in 2020, an increase of $9.68 \%$. The total number of GP Out-of-Hours contacts was 3,803,553 in 2019, and $3,665,455$ in 2020 , a decrease of $3.63 \%$. The number of GP Out-of-Hours contacts assigned a Read code was $1,634,938$ in 2019 (43\% of contacts), and 1,331,478 in
2020 (36\% of contacts). The daily mean GPIH registered practice population was 23,998,209 in 2019, and $39,455,662$ in 2020 , an increase of $64.41 \%$. The number of ambulance calls was 2,100,527 in 2019, and 2,105,082 in 2020 , a slight increase of $0.22 \%$. The total number of attendances at the 100 emergency departments included in the study was $3,909,796$ in 2019 , and $2,997,082$ in 2020 , a decrease of $23.34 \%$. The number of ED attendances with a diagnosis was 3,138,470 in 2019 (80\%), and 2,491,255 in 2020 (83\%).

Daily trends in total activity across all five syndromic surveillance systems showed significant variation during the COVID-19 pandemic in England compared to equivalent months in 2019 (Fig. 2). The daily mean percentage changes in activity during the peak pandemic period in 2020 (Weeks 13 to 22) compared to the same period in 2019 are shown in Table 1. These showed the least difference for NHS 111 calls (12\% increase; 95\%CI: 9 to $15 \%)$ and $\mathrm{GPOOH}$ consultations (11\% decrease; 95\%CI: -8 to $-14 \%)$. The total number of ambulance calls varied markedly throughout the pandemic period, increasing during the introduction of social distancing, before reducing during lockdown, such that the mean daily number during the peak pandemic period hides the marked variation seen in Fig. 2. The total number of attendances at ED nearly halved during the peak pandemic (daily mean percentage change: $-49 \%$ (95\%CI: $-45 \%$ to $-52 \%)$. (Trends in healthcare usage during the peak pandemic period in 2020 compared to 2019, stratified by weekdays, and weekend and bank holidays, for each syndromic surveillance system and indicators are available in Additional Table 1.)

Eye conditions, monitored through GPIH, GPOOH, and NHS 111, showed a sharp decline in mid-February (earlier than other syndromic indicators). GPIH conjunctivitis remained low throughout the 2020 peak pandemic period (64\% reduction; $95 \% \mathrm{CI}:-63 \%$ to $-65 \%$, compared to 2019 during peak pandemic period) as did GPOOH eye irritation (71\% reduction; $95 \% \mathrm{CI}$ : $-68 \%$ to - 74\%). In contrast, though the number of calls to NHS 111 for eye problems initially reduced during March, call numbers returned to baseline (2019) by May, resulting in a smaller (25\%; 95\%CI: $-20 \%$ to $-31 \%$ ) reduction across the entire peak pandemic period (Fig. 3).

GP In Hours mumps consultations showed a dramatic decline after the introduction of NPIs, following high activity early in 2020. During the peak pandemic period, the number of GP In Hours consultations for mumps showed a $31 \%(95 \% \mathrm{CI}$ : $-11 \%$ to $-54 \%)$ reduction compared to 2019 (Fig. 4).

Fractures presenting to EDs showed a sharp decline from the 12th March 2020 when COVID-19 restrictions were introduced, before starting to increase during May (Fig. 5a). During the peak pandemic period, there was a 

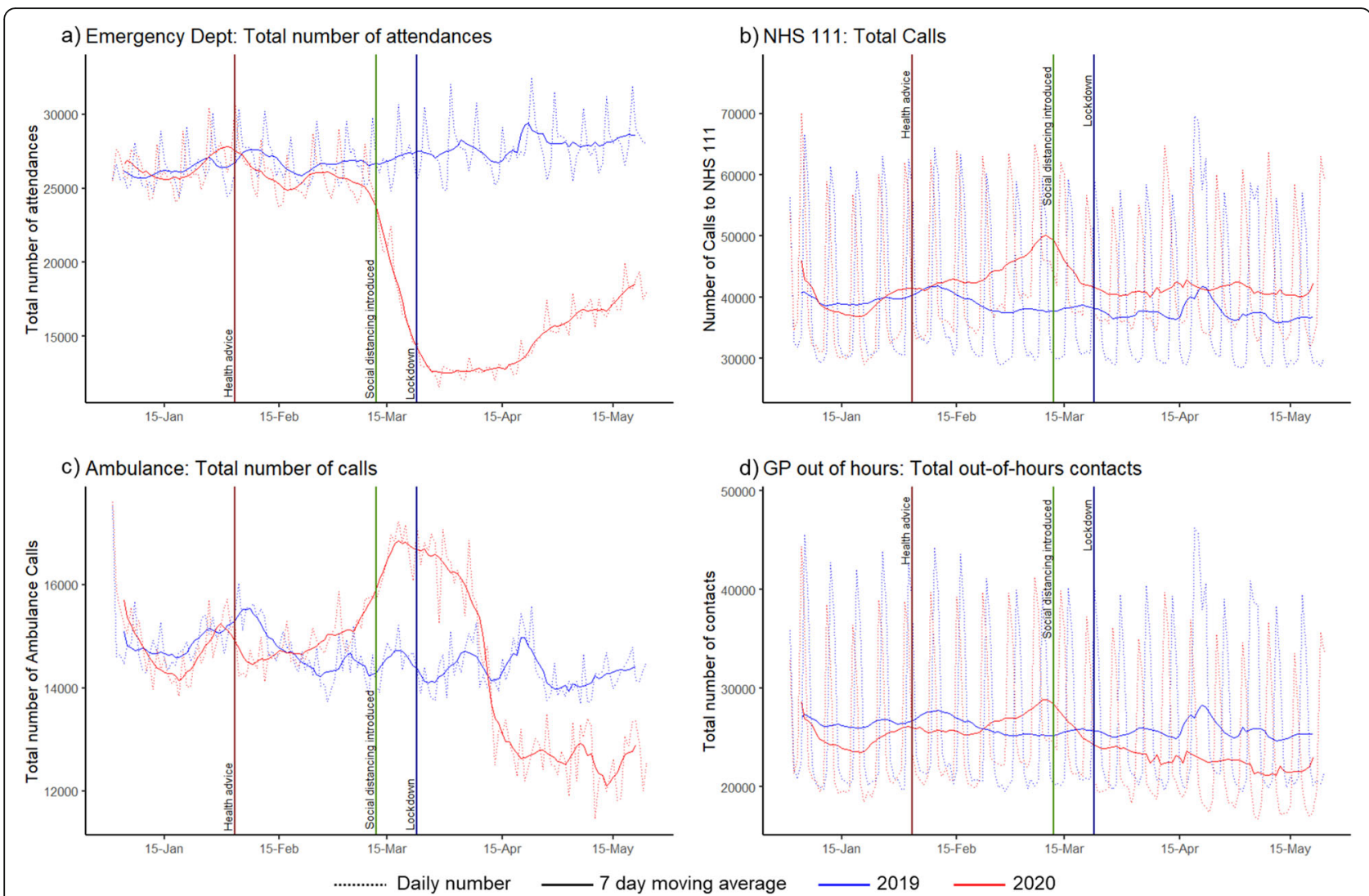

Fig. 2 Overall total numbers of contacts in four different syndromic surveillance systems. These graphs show the daily (dotted line) and 7-day moving average (solid line) a) total number of attendances at emergency departments, b) total number of calls to NHS 111 as rate per 100,000 population, c) total number of calls to 999 for an ambulance, and d) total number of contacts with GP Out-of-Hours services

$49 \%$ (95\%CI: $-46 \%$ to $-52 \%)$ reduction in attendance at ED for fractures compared to the same period in 2019. In 2019, weeks 13 to 22, the mean number of females attending ED with a fracture was highest on Monday mornings and for males there were peaks on both Sunday late morning and Monday morning. The trends of ED attendances by hour of day and day of week for the peak pandemic period in 2020 demonstrated that there was no longer any weekend effect, resulting in similar patterns of attendance across each day of the week for both males and females (Fig. 5b, c).

GP In Hours consultations for herpes zoster showed a decline following the interventions in March and an overall $27 \%$ reduction $(95 \% \mathrm{CI}$ : $-23 \%$ to $-31 \%)$ in consultations during the peak pandemic period compared to 2019 (Fig. 4).

ED attendances for myocardial ischaemia also showed a marked decline following the interventions in March, with a $35 \%$ reduction $(95 \% \mathrm{CI}:-31 \%$ to $-39 \%)$ in attendance for the peak pandemic period in 2020 compared to 2019 (Fig. 6). Opposing trends were observed for chest pain in ambulance calls and chest pain/myocardial infarction in $\mathrm{GPOOH}$.

\section{Discussion}

There were significant changes to healthcare utilisation in the first few months of the pandemic response in England. Total numbers of contacts with healthcare services in England, as demonstrated through the NHS 111, Ambulance, and both GP syndromic surveillance systems, increased in early March. A marked decline in activity, compared to 2019, was then observed across all syndromic systems (including ED) around the time of the introduction of COVID-19 NPIs and restrictions to activities in mid to late March. Similar rapid reductions in ED visits have also been reported in the USA, where ED visits declined by $42 \%$ during the early COVID- 19 pandemic period, particularly among children and females, and in Thailand where ED visits overall declined by $36 \%$ [31, 32]. A systematic review summarising the impact of COVID-19 pandemic on healthcare utilisation from 81 studies across 20 countries published before 10 August 2020 found a median 37\% reduction in services overall compared to pre-pandemic trends [33].

Healthcare contacts for different conditions displayed different trends during the peak pandemic period. Eye conditions showed a decline in activity earlier than other 

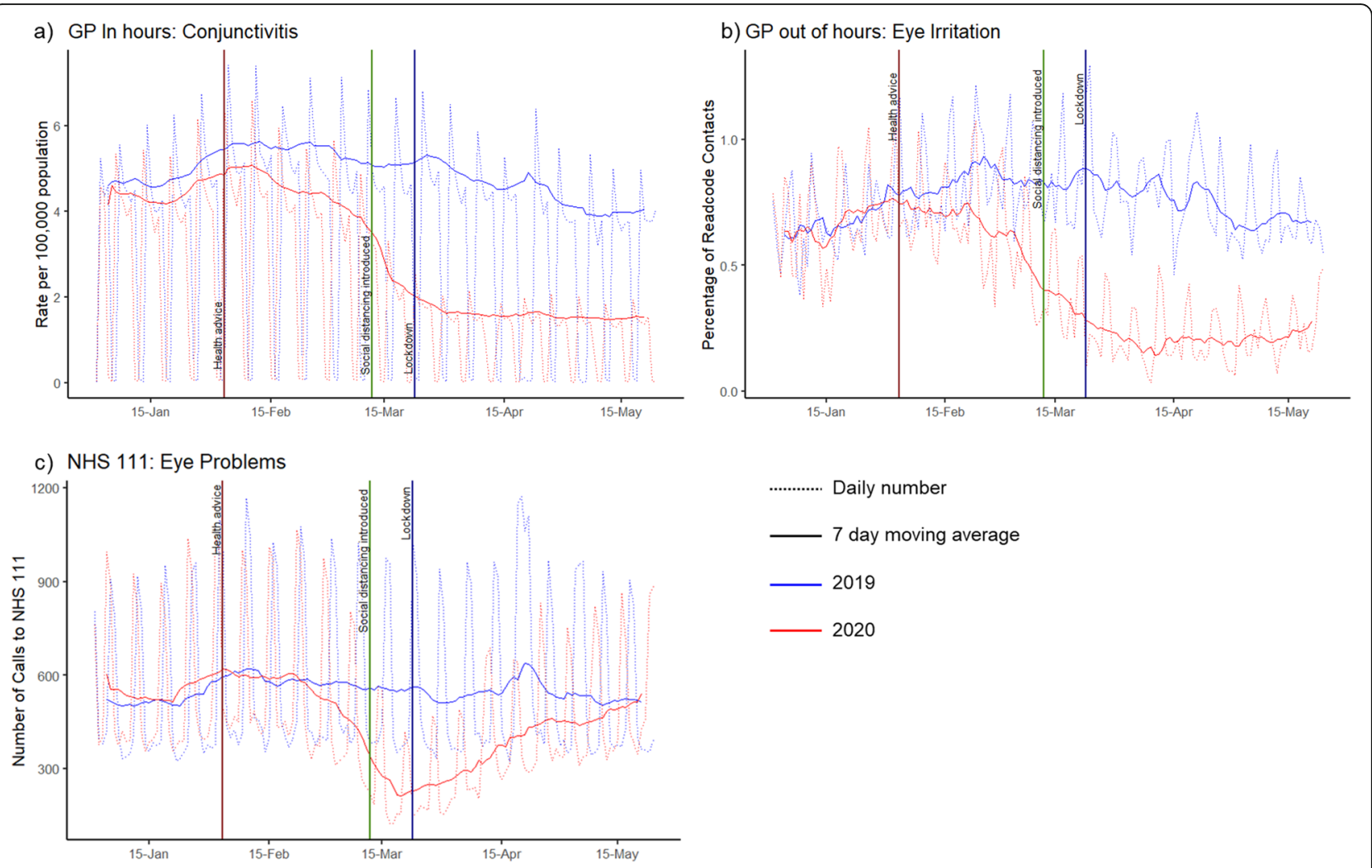

Fig. 3 Numbers of contacts for eye conditions in three different syndromic surveillance systems. These graphs show the daily (dotted line) and 7-day moving average (solid line) a) number of GP In Hours consultations for 'conjunctivitis' per 100,000 population, b) number of GP Out-of-hours contacts for 'eye irritation' as a percentage of contacts with a Read code and c) number of calls per 100,000 population to NHS 111 for 'eye problems'

indicators, which may be explained by healthcare utilisation changing most significantly for milder conditions, or this may reflect a true decrease in incidence due to changes in hygiene behaviour reducing the spread of infectious conjunctivitis [23]. The persistence of low activity of eye conditions may be due to the closure of schools and nurseries during the peak pandemic period. Improvements in hygiene behaviour, combined with social distancing and in particular the closure of universities in mid-March, may also explain the dramatic reduction in mumps consultations in GPIH, bringing a rapid end to the national mumps outbreak of 2019/2020

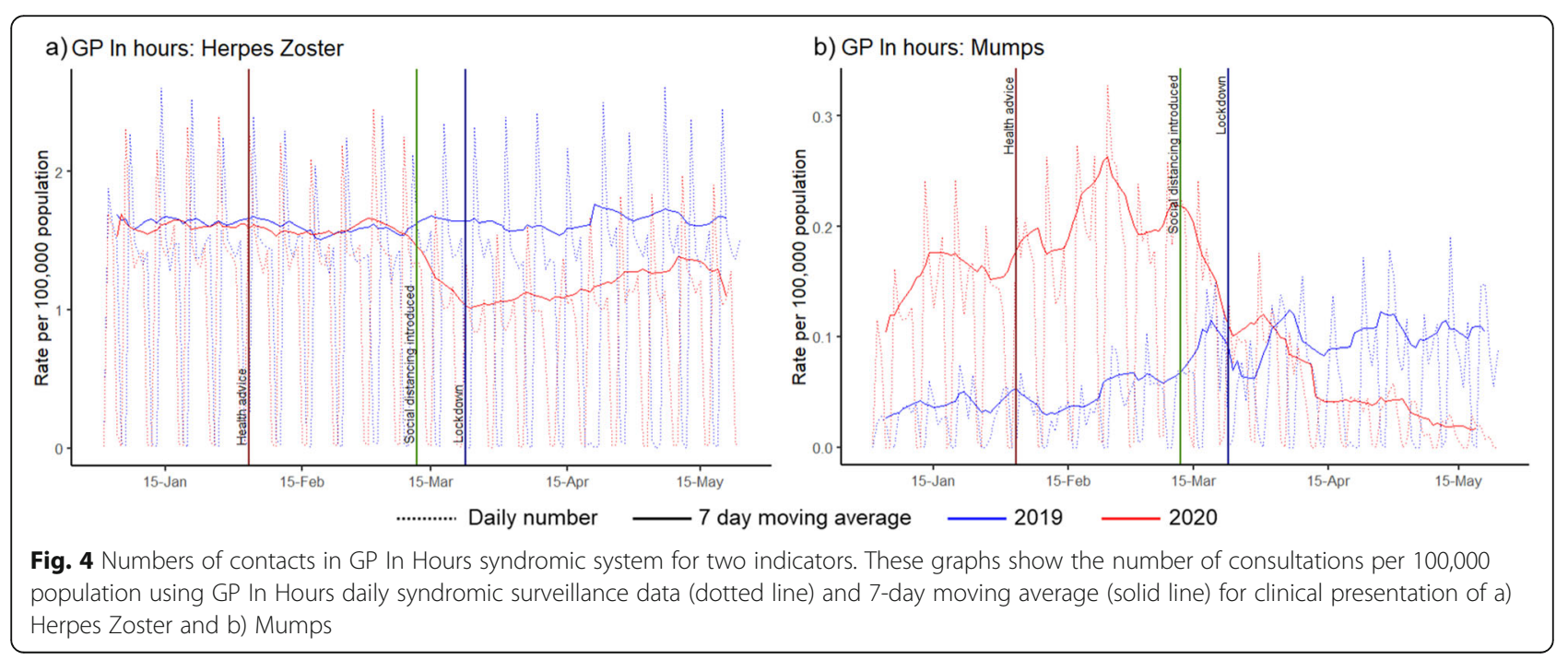




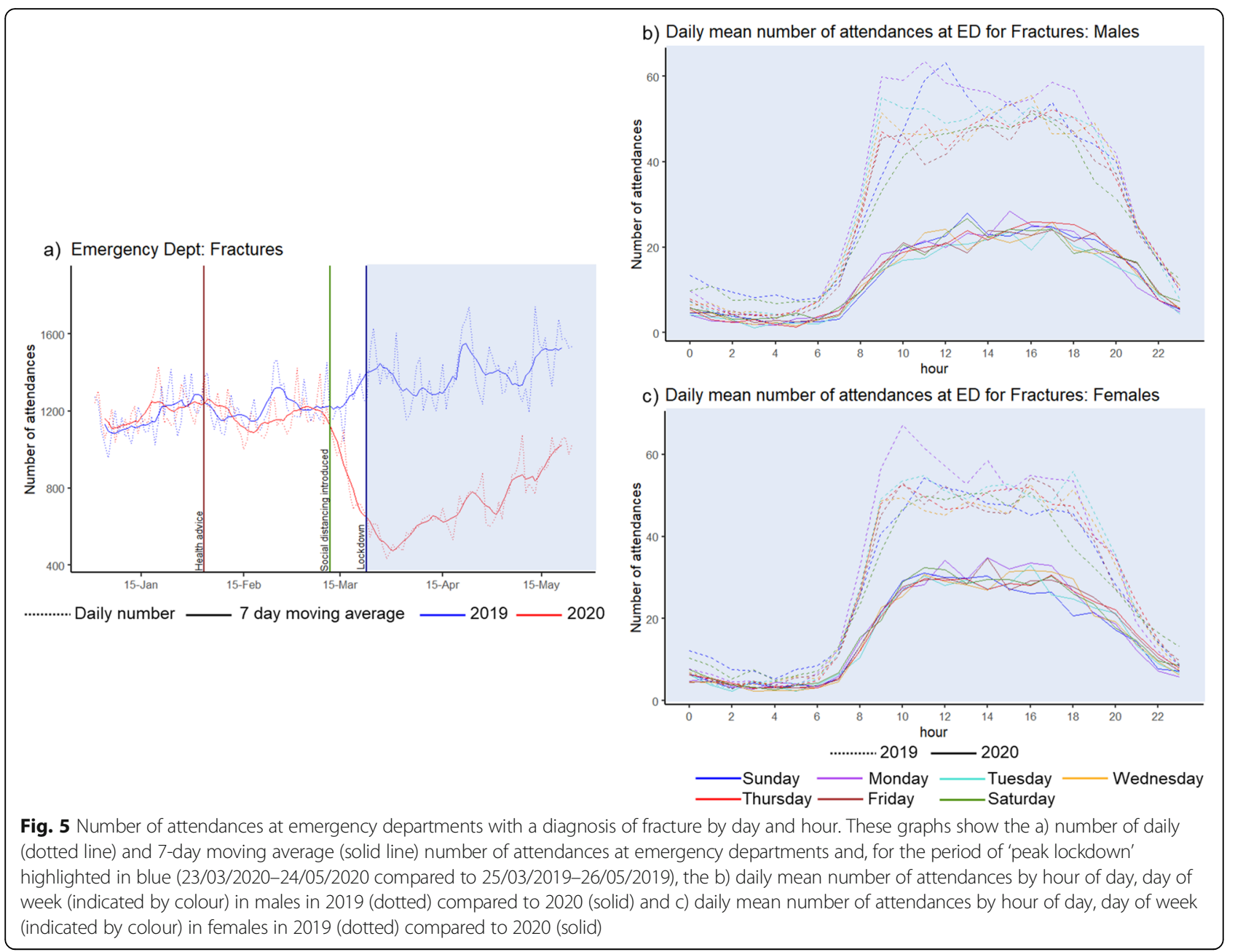

that was concentrated particularly in university student populations [24].

The trends in attendances at ED for fractures, a high acuity condition that is more likely to represent true incidence, showed overall reduced numbers during the peak pandemic study period. Other data sources for incidence of trauma during COVID-19 show the same trend: referrals to specialist spinal surgery decreased significantly compared to the same period in 2019, most apparent for the number of high-energy traumatic presentations, which declined by $72 \%(p<0.002)$ [34]. This is likely because of significant changes to daily activities, including the closing of schools and less participation in team sports, during the peak pandemic period. Data from the UK Government Department for Transport show mean car traffic use during the peak pandemic (weeks 13 to 22 ) was reduced by $61 \%$ compared to the baseline first week of February 2020 [35] likely resulting in fewer road traffic collisions. The trends in attendances for fractures show a loss of the weekend effect which may be partly due to a reduction in team sports and a reduction in alcohol-related attendances, as a consequence of the closure of the service industry, which has previously been shown to account for a peak of $72 \%$ of attendances in males in early hours at weekends [36]. The significant decline in ED attendance due to myocardial ischaemia during the COVID-19 pandemic was of concern to clinicians especially given the association between acute infection and myocardial infarction [37]. Further analysis of ED data showed the reduction in attendance was greatest at lower levels of acuity, and yet level 1 acuity 'immediate' attendances still reduced by $31 \%$ during COVID-19 [10]. This is in keeping with other reports on the reduction in the number of stroke patients attending, where the greatest decline was among mild severity stroke [38] and systematic review finding larger reductions in utilisation among people with milder spectrum of illness [33]. These dramatic declines in attendances at ED prompted public health messaging urging patients to continue to seek medical care as required [39].

A strength of this study is its use of the breadth of data across five national syndromic surveillance systems 

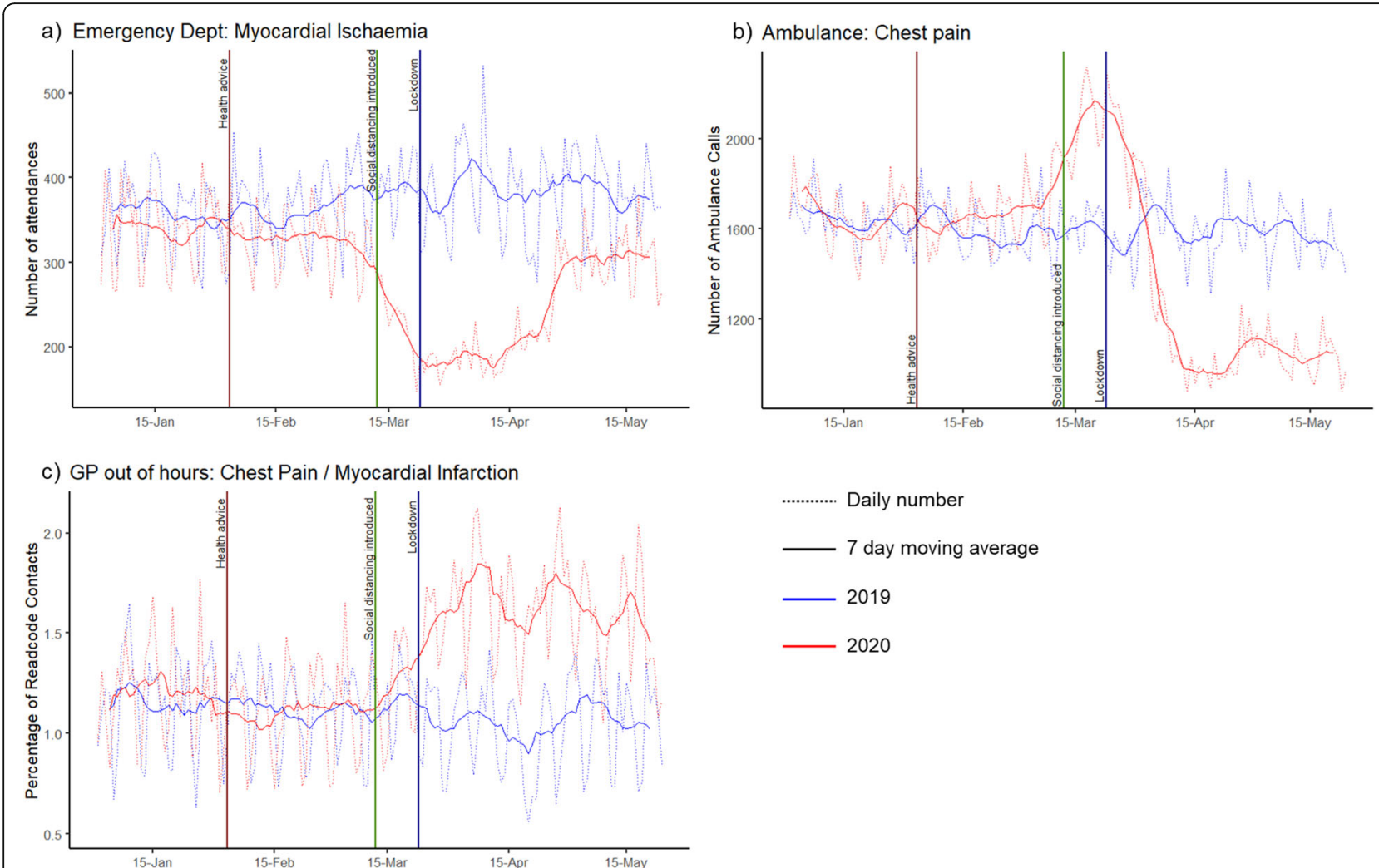

Fig. 6 Numbers of contacts for chest-pain syndromes in three different syndromic surveillance systems. These graphs show daily syndromic surveillance data (dotted line) and 7-day moving average (solid line) for a) number of attendances with 'Myocardial Ischaemia' at emergency departments b) number of calls to 999 for an ambulance due to 'Chest Pain', and c) number of GP Out-of-Hours contacts for 'Chest Pain / Myocardial Infarction' as a percentage of contacts with a Read code

to demonstrate changes in healthcare utilisation across several healthcare systems and for a wide range of mild to severe conditions in England over time. This routine, real-time monitoring of syndromic indicators of disease provides a greater understanding of trends in healthcare utilisation, particularly for infectious diseases [13, 40]. The ability of ED data to add insight into changing trends in attendances by hour of day and day of week provides an added benefit of working with this syndromic data. This has previously been used to analyse the impact of sporting events on the timing of ED attendances $[18,41]$.

One limitation of the study is that some changes in indicator trends may result from modifications that have been made to the underlying health care systems to improve patient management rather than actual changes in activity. However, experience and detailed knowledge of the surveillance system and data by those undertaking the surveillance can help to minimise these limitations. For example, opposing trends in chest pain syndromes were observed throughout the first lockdown, however, the marked reduction in chest pain in the Ambulance system followed the introduction of a 'pandemic' chief complaint pathway (the basis of a newly defined COVID-19-like syndromic indicator) in early March. This suggested that the earlier peak in chest pain may have been detecting increased incidence of COVID-19 and/or anxiety, rather than cardiac-related chest pain. A COVID-19-like indicator was not introduced in the $\mathrm{GPOOH}$ system and therefore the chest pain/myocardial infarction indicator may have continued to capture some COVID-19 activity (albeit a small increase in percentage of Read code consultations). This demonstrates the importance of detailed interpretation from experts who understand the data's strengths and limitations. Additionally, it is difficult to disentangle behavioural changes in healthcare usage and changes in disease incidence. This was in part minimised by exploring a range of different surveillance systems and indicators, for both mild and more severe health conditions.

This study improves our understanding of how healthcare usage changed during the first national lockdown, which began on the 23rd March 2020, both in total and for a range of syndromic indicators. This understanding is key when interpreting syndromic surveillance data and considering healthcare utilisation during the second 
and third national lockdowns (which were introduced in November 2020 and January 2021 respectively), when the NHS continued much of its routine healthcare provision and public health messaging about the importance of attending healthcare when needed was generally better emphasised [42]. Future work could describe how trends in syndromic indicators changed throughout summer 2020 when fewer restrictions were in place. $\mathrm{Fu}$ ture sub-analyses could include exploring the trends in attendances for fractures by gender and age-groups and myocardial ischaemia by severity of illness markers. Importantly, our increased knowledge of how trends in syndromic surveillance are changing throughout the COVID-19 pandemic will be important when interpreting trends in other conditions, for example the monitoring of influenza throughout the seasonally expected peak influenza activity season, against a background of changing healthcare usage, behaviours, and incidence of other conditions.

\section{Conclusions}

This descriptive analysis of syndromic surveillance system data demonstrates the significant impact of COVID-19 on England's health, National Health Service and society. The restrictions introduced to contain and delay the spread of COVID-19 and to ease pressure on the health service resulted in a combination of a likely true decrease in incidence and a change in healthcare seeking behaviour for certain conditions. Syndromic surveillance systems have a valued and important role in describing and interpreting these trends.

\section{Abbreviations}

NHS: National Health Service; PHE: Public Health England; GP: General Practitioner; GPIH: GP In Hours; GPOOH: GP Out-of-Hours; ED: Emergency Department; NPIs: Non-pharmaceutical interventions; BCa: bias corrected and accelerated

\section{Supplementary Information}

The online version contains supplementary material available at https://doi. org/10.1186/s12889-021-12117-5.

Additional file 1: Table S1. Daily mean percentage change between 2019 and 2020 peak pandemic period by indicators.

\section{Acknowledgements}

The authors acknowledge the contribution and support from all data providers including:

- NHS 111 and NHS Digital.

- QSurveillance ${ }^{\oplus}$; University of Oxford; EMIS/EMIS practices; ClinRisk ${ }^{\bullet}$.

- TPP, ResearchOne and participating SystmOne GP practices.

- Advanced Health \& Care and the participating $\mathrm{OOH}$ service providers.

- Participating ED syndromic surveillance system emergency departments.

- Royal College of Emergency Medicine.

- North East, North West, Yorkshire, East Midlands, West Midlands, East of England, London, South East Coast, South Central, and South Western NHS Ambulance Trusts and The Association of Ambulance Chief Executives.

\section{Authors' contributions}

Conceptualisation: CFF, LF, HEH, GES, IO; methodology: CFF, LF, HEH, IO, RM GES; formal analysis: CFF, LF, RM, HEH; investigation: CFF, LF, RM; resources and data curation: RM, GES, HEH, AJE, SH, TCH; writing - original draft preparation: CFF, LF; writing - review and editing: all authors; visualisation: CFF, RM; supervision: HEH, GES, IO; project administration: CFF, LF; funding acquisition: IO, GES. All authors have read and agreed to the final published version of the manuscript.

\section{Funding}

CFF, LF and IO receive support from the National Institute for Health Research Health Protection Research Unit (NIHR HPRU) in Behavioural Science and Evaluation. GES, RM and AJE receive support from the NIHR HPRU in Emergency Preparedness and Response. GES, HEH and AJE receive support from the NIHR HPRU in Gastrointestinal Infections.

The views expressed are those of the author(s) and not necessarily those of the NIHR, Public Health England or the Department of Health and Social Care.

\section{Availability of data and materials}

The datasets analysed in this study are not publicly available. Syndromic surveillance data are collected and held by PHE for surveillance purposes, with no provision for use other than national surveillance outputs. Further information about accessing PHE data through the Office for Data Release can be found here: https://www.gov.uk/government/publications/accessingpublic-health-england-data/about-the-phe-odr-and-accessing-data

\section{Declarations}

Ethics approval and consent to participate

The anonymised health data used in this study were routinely collected as part of the public health function of PHE. PHE has access to a range of data sources under Regulation 3 (Health Protection) of The Health Service (Control of Patient Information) Regulations 2002. Informed consent from individuals was not required.

This study was undertaken as part of the national surveillance functions of Public Health England, in accordance with relevant guidelines and regulations. The UK Health Research Authority decision tool defined this study as "Usual Practice" (http://www.hra-decisiontools.org.uk/ research/docs/ DefiningResearchTable_Oct2017-1.pdf) as such ethical approval for this work was not required. All experimental protocols were approved by Public Health England.

Consent for publication

Not applicable.

\section{Competing interests}

$\mathrm{TCH}$ is a director of $\mathrm{L} 2 \mathrm{~S} 2 \mathrm{Ltd}$. All other authors declare that they have no competing interests.

\section{Author details}

${ }^{1}$ Field Service, UK Health Security Agency, Bristol BS1 6EH, UK. ${ }^{2}$ National Institute of Health Research Health Protection Research Unit on Behavioural Science and Evaluation at the University of Bristol, Bristol, UK. ${ }^{3}$ Real-time Syndromic Surveillance Team, Field Service, UK Health Security Agency, Birmingham B3 2PW, UK. ${ }^{4}$ John Radcliffe Hospital, Oxford, Oxfordshire, UK.

Received: 25 January 2021 Accepted: 29 September 2021

Published online: 05 November 2021

\section{References}

1. World Health Organisation. [Internet] WHO Coronavirus Disease (COVID-19) Dashboard. 2020. Geneva, Switzerland. Available from: https://covid19.who. int/ Accessed $21^{\text {st }}$ June 2021.

2. Lillie PJ, Samson A, Li A, Adams K, Capstick R, Barlow GD, et al. Novel coronavirus disease (COVID-19): the first two patients in the UK with person to person transmission. J Inf Secur. 2020;80(5):578-606. https://doi.org/10.1 016/j.jinf.2020.02.020.

3. Spiteri G, Fielding J, Diercke M, Campese C, Enouf V, Gaymard A, et al. First cases of coronavirus disease 2019 (COVID-19) in the WHO European Region, 24 January to 21 February 2020. Euro Surveill. 2020;25(9):2000178. 
4. Public Health England. [Internet] Weekly Coronavirus Disease 2019 (COVID19) Surveillance Report - Week 35. Available from: https://www.gov.uk/ government/news/weekly-covid-19-surveillance-report-published Accessed 2nd September 2020.

5. UK Government. [Internet] Coronavirus public information campaign launched across the UK. London, England. Available from: https:/www.gov. uk/government/news/coronavirus-public-information-campaign-launched-a cross-the-uk Accessed 2nd September 2020.

6. UK Government. [Internet] Prime Minister's statement on coronavirus (COVID-19): 12 March 2020 London, England. Available from: https://www. gov.uk/government/speeches/pm-statement-on-coronavirus-12-march-2020 Accessed 2nd September 2020.

7. NHS. NHS 111 Online Get medical help near you. [Internet] England. Available from: www.111.nhs.uk Accessed 2nd September 2020.

8. Greenhalgh T, Koh GCH, Car J. COVID-19: a remote assessment in primary care. BMJ. 2020;368:m1182. https://doi.org/10.1136/bmj.m1182.

9. UK Government. [Internet] Coronavirus (COVID-19) in the UK. London, England. Available from: www.coronavirus.data.gov.uk2020 Accessed 2nd September 2020.

10. Hughes HE, Hughes TC, Morbey R, Challen K, Oliver I, Smith GE, et al. Emergency department use during COVID-19 as described by syndromic surveillance. Emerg Med J. 2020;37(10):600-4. https:/doi.org/10.1136/ emermed-2020-209980.

11. Public Health England. [Internet] National COVID-19 surveillance report. Available from: https://www.gov.uk/government/publications/nationalcovid-19-surveillance-reports Accessed 2nd October 2020.

12. Triple S Project. Assessment of syndromic surveillance in Europe. Lancet. 2011;378(9806):1833-4. https://doi.org/10.1016/S0140-6736(11)60834-9.

13. Harcourt SE, Smith GE, Elliot AJ, Pebody R, Charlett A, lbbotson S, et al. Use of a large general practice syndromic surveillance system to monitor the progress of the influenza a(H1N1) pandemic 2009 in the UK. Epidemiol Infect. 2012;140(1):100-5. https://doi.org/10.1017/S095026881100046X.

14. Chu A, Savage R, Willison D, Crowcroft NS, Rosella LC, Sider D, et al. The use of syndromic surveillance for decision-making during the H1N1 pandemic: a qualitative study. BMC Public Health. 2012;12(1):929. https:/doi.org/10.11 86/1471-2458-12-929.

15. Smith S, Elliot AJ, Hajat S, Bone A, Bates C, Smith GE, et al. The Impact of Heatwaves on Community Morbidity and Healthcare Usage: A Retrospective Observational Study Using Real-Time Syndromic Surveillance. Int J Environ Res Public Health. 2016;13(1):132.

16. Smith S, Elliot AJ, Hajat S, Bone A, Smith GE, Kovats S. Estimating the burden of heat illness in England during the 2013 summer heatwave using syndromic surveillance. J Epidemiol Community Health. 2016;70(5):459-65. https://doi.org/10.1136/jech-2015-206079.

17. Hughes HE, Morbey R, Hughes TC, Locker TE, Shannon T, Carmichael C, et al. Using an emergency department syndromic surveillance system to investigate the impact of extreme cold weather events. Public Health. 2014; 128(7):628-35. https://doi.org/10.1016/j.puhe.2014.05.007.

18. Smith GE, Elliot AJ, Ibbotson S, Morbey R, Edeghere O, Hawker J, et al. Novel public health risk assessment process developed to support syndromic surveillance for the 2012 Olympic and Paralympic games. J Public Health (Oxf). 2017;39(3):e111-e7. https://doi.org/10.1093/pubmed/fdw054.

19. Elliot AJ, Harcourt SE, Hughes HE, Loveridge P, Morbey RA, Smith S, et al. The COVID-19 pandemic: a new challenge for syndromic surveillance. Epidemiol Infect. 2020;148:e122. https://doi.org/10.1017/S095026882 0001314.

20. National Health Service [Internet] Monthly A\&E Attendances and Emergency Admisisons 2019-20 and 2020-21. https://www.england.nhs.uk/statistics/sta tistical-work-areas/ae-waiting-times-and-activity/2020 Accessed $21^{\text {st }}$ December 2020

21. Harcourt SE, Morbey RA, Loveridge P, Carrilho L, Baynham D, Povey E, et al. Developing and validating a new national remote health advice syndromic surveillance system in England. J Public Health (Oxf). 2017;39(1):184-92.

22. Elliot AJ, Hughes HE, Hughes TC, Locker TE, Shannon T, Heyworth J, et al. Establishing an emergency department syndromic surveillance system to support the London 2012 Olympic and Paralympic games. Emerg Med J. 2012;29(12):954-60. https://doi.org/10.1136/emermed-2011-200684.

23. Kim HS, Choi HC, Cho B, Lee JY, Kwon MJ. Effect of the H1N1 influenza pandemic on the incidence of epidemic keratoconjunctivitis and on hygiene behavior: a cross-sectional study. PLoS One. 2011;6(8):e23444. https://doi.org/10.1371/journal.pone.0023444.
24. Public Health England. [Internet] Mumps outbreaks across England Available from: https://www.gov.uk/government/news/mumps-outbreaks-acrossengland Accessed 2nd September 2020.

25. de Lusignan S. Codes, classifications, terminologies and nomenclatures: definition, development and application in practice. Inform Prim Care. 2005; 13(1):65-70. https://doi.org/10.14236/jhi.v13i1.580.

26. Buckingham-Jeffery E, Morbey R, House T, Elliot AJ, Harcourt S, Smith GE. Correcting for day of the week and public holiday effects: improving a national daily syndromic surveillance service for detecting public health threats. BMC Public Health. 2017;17(1):477. https://doi.org/10.1186/s12889-01 7-4372-y.

27. Wickham H, Averick M, Bryan J, Chang W, D'Agostino McGowan L, Francois R, et al. Welcome to the tidyverse. J Open Source Softw. 2019;4(43):1686. https://doi.org/10.21105/joss.01686.

28. Davison AC, Hinkley DV. Bootstrap methods and their applications. Cambridge: Cambridge University Press; 1997. https://doi.org/10.1017/ CBO9780511802843.

29. Canty A, Ripley B. boot: Bootstrap R (S-Plus) Functions. R package version 1. 2020:3-25.

30. Wickham H. ggplot2: Elegant Graphics for Data Analysis. New York: Springer-Verlag; 2016.

31. Hartnett KP, Kite-Powell A, DeVies J, Coletta MA, Boehmer TK, Adjemian J, et al. Impact of the COVID-19 pandemic on emergency department visits United States, January 1, 2019-may 30, 2020. MMWR Morb Mortal Wkly Rep. 2020;69(23):699-704. https://doi.org/10.15585/mmwr.mm6923e1.

32. Wongtanasarasin $W$, Srisawang $T$, Yothiya W, Phinyo P. Impact of national lockdown towards emergency department visits and admission rates during the COVID-19 pandemic in Thailand: a hospital-based study. Emerg Med Australasia. 2020;33(2):316-23. https://doi.org/10.1111/1742-6723.13666.

33. Moynihan R, Sanders S, Michaleff ZA, Scott AM, Clark J, To EJ, et al. Impact of COVID-19 pandemic on utilisation of healthcare services: a systematic review. BMJ Open. 2021;11(3):e045343. https://doi.org/10.1136/bmjopen-202 0-045343.

34. Ahuja S, Shah P, Mohammed R. Impact of COVID-19 pandemic on acute spine surgery referrals to UK tertiary spinal unit: any lessons to be learnt? $\mathrm{Br}$ J Neurosurg. 2020;35(2):1-5. https://doi.org/10.1080/02688697.2020.1777263.

35. UK Government. [Internet] Transport use during the coronavirus (COVID-19) pandemic. Department for Transport. London, England. Available from: https://www.gov.uk/government/statistics/transport-use-during-the-corona virus-covid-19-pandemic Accessed 21st October 2020.

36. Parkinson K, Newbury-Birch D, Phillipson A, Hindmarch P, Kaner E, Stamp E, et al. Prevalence of alcohol related attendance at an inner city emergency department and its impact: a dual prospective and retrospective cohort study. Emerg Med J. 2016;33(3):187-93. https://doi.org/10.1136/emermed-2 014-204581

37. Musher DM, Abers MS, Corrales-Medina VF. Acute infection and myocardial infarction. N Engl J Med. 2019;380(2):171-6. https://doi.org/10.1056/NEJMra1 808137.

38. Perry R, Banaras A, Werring DJ, Simister R. What has caused the fall in stroke admissions during the COVID-19 pandemic? J Neurol. 2020;267(12):3457-8. https://doi.org/10.1007/s00415-020-10030-2.

39. NHS [Internet] Help us help you: NHS urges public to get care when they need it England. Available from: https://www.england.nhs.uk/2020/04/helpus-help-you-nhs-urges-public-to-get-care-when-they-need-it/ Accessed 2nd September 2020.

40. Bawa Z, Elliot AJ, Morbey RA, Ladhani S, Cunliffe NA, O'Brien SJ, et al. Assessing the likely impact of a rotavirus vaccination program in England: the contribution of syndromic surveillance. Clin Infect Dis. 2015;61(1):77-85. https://doi.org/10.1093/cid/civ264.

41. Hughes HE, Colon-Gonzalez FJ, Fouillet A, Elliot AJ, Caserio-Schonemann C, Hughes TC, et al. The influence of a major sporting event upon emergency department attendances; a retrospective cross-national European study. PLoS One. 2018;13(6):e0198665. https://doi.org/10.1371/journal.pone.0198665.

42. UK Government. [Internet] Prime Minister's statement on coronavirus, 31st October 2020. London, England. Available from: https:/www.gov.uk/ government/speeches/prime-ministers-statement-on-coronavirus-covid-1 9-31-october-2020 Accessed 11th November 2020.

\section{Publisher's Note}

Springer Nature remains neutral with regard to jurisdictional claims in published maps and institutional affiliations. 